\title{
Long-Term Effects of Ipragliflozin on Diabetic Nephropathy and Blood Pressure in Patients With Type 2 Diabetes: 104- Week Follow-up of an Open-Label Study
}

\author{
Daisuke Ito $^{\text {a, b, f , Kazuyuki Inoue }}{ }^{\mathrm{a}}$, Takashi Sumita ${ }^{\mathrm{a}, \mathrm{b}}$, Keiko Hamaguchic $^{\mathrm{c}}$, Kimie Kaneko ${ }^{\mathrm{c}}$, \\ Morifumi Yanagisawa ${ }^{\mathrm{d}}$, Kouichi Inukaie, Ikuo Inoue ${ }^{\mathrm{a}}$, \\ Mitsuhiko Noda ${ }^{\text {a }}$, Akira Shimada ${ }^{\mathrm{a}}$
}

\begin{abstract}
Background: In our previous study, we investigated the efficacy of ipragliflozin, a sodium-glucose cotransporter (SGLT) 2 inhibitor on diabetic nephropathy in patients with type 2 diabetes and demonstrated that ipragliflozin significantly improved diabetic nephropathy in addition to reducing HbAlc and body weight. Herein, we conducted post-trial monitoring to determine whether these lowering effects on blood glucose and body weight or the beneficial effects on diabetic nephropathy were maintained long-term (104 weeks) after starting ipragliflozin treatment.
\end{abstract}

Methods: Initially, during a 24-week interventional trial period, a 50 $\mathrm{mg}$ dose of ipragliflozin was administered to 50 patients with type 2 diabetes without changing other treatments. During the post-trial monitoring period, these patients returned to hospital-based diabetes care according to their clinical needs. We continued monitoring their clinical data for 104 weeks in each hospital and analyzed the results on an intention-to-treat basis.

Results: The improvements in glycemic control and body weight reduction provided by 24 -week ipragliflozin administration were maintained for 104 weeks. Despite a transient decrease during the intervention period, the estimated glomerular filtration rate (eGFR) was restored to near the baseline level at 104 weeks. Notably, in patients with diabetic nephropathy, the median urinary albumin-to-creatinine ratio (UACR) was significantly decreased from 119.2 (98.9 - 201.8) at baseline to $36.9(19.7-204.7) \mathrm{mg} / \mathrm{gCr}$ at 104 weeks. In addition,

Manuscript submitted May 27, 2018, accepted June 27, 2018

aDepartment of Endocrinology and Diabetes, Saitama Medical University, Saitama, Japan

${ }^{b}$ Department of Internal Medicine, Ogawa Red Cross Hospital, Saitama, Japan 'Department of Nursing, Ogawa Red Cross Hospital, Saitama, Japan

dSatsuki Medical Clinic, Saitama, Japan

eDepartment of Diabetes and Endocrinology, Higashiyamato Hospital, Tokyo, Japan

${ }^{\mathrm{f} C}$ Corresponding Author: Daisuke Ito, Department of Endocrinology and Diabetes, Saitama Medical University, 38, Morohongo, Moroyama, Iruma-gun, Saitama, 350-0495, Japan. Email: dito@saitama-med.ac.jp

doi: https://doi.org/10.14740/jocmr3491w
eGFR was stable for 104 weeks, showing no decrease. In contrast, a significant positive correlation between UACR and blood pressure observed at 24 weeks disappeared after discontinuation of the intervention therapy.

Conclusions: The well-controlled $\mathrm{HbA} 1 \mathrm{c}$ and body weight reductions were maintained for 104 weeks of post-trial follow-up. Moreover, ipragliflozin significantly reduced urinary albumin excretion in patients with diabetic nephropathy without decreasing eGFR.

Keywords: Ipragliflozin; Sodium-glucose cotransporter 2 inhibitor; Diabetic nephropathy; Blood pressure; Type 2 diabetes; Long-term

\section{Introduction}

Sodium-glucose cotransporter (SGLT) 2 inhibitors reduce rates of hyperglycemia in patients with diabetes by decreasing renal glucose reabsorption, thereby increasing urinary glucose excretion [1]. In addition to their glucose-lowering actions, SGLT2 inhibitors exert favorable effects on various biomarkers, including body weight [2-4], blood pressure [5], albuminuria [6], and fatty liver [7], as well as also possibly reducing the risk of serious cardiovascular complications, diabetic kidney disease, and death [8-10]. In the EMPA-REG OUTCOME trial, empagliflozin, an SGLT2 inhibitor, reduced the risk of major adverse cardiovascular events in patients with type 2 diabetes at high risk for such events [9]. In the sub-analysis of the EMPA-REG OUTCOME trial that evaluated renal events, empagliflozin was associated with slower progression of kidney disease and lower rates of clinically relevant renal events [10]. Although SGLT2 inhibitors are highly anticipated to exert renoprotective effects, the question of whether improved glucose control persisted and whether such therapy exerted a long-term effect on diabetic nephropathy and blood pressure in routine clinical practice remains unclear.

Our previous study, an open-label, 24-week, prospective, multicenter trial, investigated the efficacy of ipragliflozin, an SGLT2 inhibitor, on diabetic nephropathy and blood pressure in patients with type 2 diabetes (UMIN Clinical Trials Registry, number UMIN000022615) [11]. The study demonstrated 
that ipragliflozin significantly reduced the median urinary albumin-to-creatinine ratio (UACR). In addition, $\mathrm{HbAlc}$ and body weight were also significantly decreased. Sub-analysis by renal function at baseline revealed the estimated glomerular filtration rate (eGFR) to be significantly decreased only in patients with apparent glomerular hyperfiltration. In contrast, although no significant changes in blood pressure levels were observed in the previous study, subgroup analysis of patients with poorly controlled blood pressure, showed significant decreases in both systolic blood pressure (SBP) and diastolic blood pressure (DBP) following 24 weeks of treatment with ipragliflozin. Furthermore, the change in UACR revealed a significant positive correlation with that in blood pressure at 24 weeks, independently of the presence of diabetic nephropathy [11].

We report herein the results of a 104-week, post-interventional follow-up of our previous study examining whether the lowering effects on blood glucose and body weight with ipragliflozin persisted after the intervention therapy completion and whether ipragliflozin had a long-term beneficial effect on diabetic nephropathy.

\section{Materials and Methods}

\section{Patients}

The study protocol, patient recruitment and methods for the intervention study were reported previously [11]. This study included participants from three hospitals in Japan and was conducted between May 2014 and April 2016, based on the ethical principles outlined in the Declaration of Helsinki. Informed consent was obtained from all patients.

Patients were between 20 and 75 years of age, with $\mathrm{HbA} 1 \mathrm{c}$ (NGSP) values of 6.5-11.0\% and a body mass index (BMI) of $22-45 \mathrm{~kg} / \mathrm{m}^{2}$. Patients with type 2 diabetes managed with diet and exercise therapy alone or antidiabetic drugs other than SGLT2 inhibitors, with or without insulin, were eligible to participate in this study. The main exclusion criteria included history of serious diabetic complications, findings suggestive of insulin dependency, heart failure (New York Heart Association Class III or IV), history of myocardial or cerebral infarction, eGFR $<45 \mathrm{~mL} / \mathrm{min} / 1.73 \mathrm{~m}^{2}$, serum creatinine $\geq 1.5 \mathrm{mg} / \mathrm{dL}$, and hepatic dysfunction.

\section{Post-trial monitoring}

All patients were enrolled in post-trial monitoring after the intervention study closed in September 2016. Patients returned to hospital-based diabetes care according to their clinical needs, with no attempt to maintain ipragliflozin administration. They were followed for 104 weeks in each hospital or clinic, with ongoing collection of outcome data; measurements of body weight, blood pressure, HbA1c, fasting plasma glucose, fasting plasma insulin, UACR, eGFR, aspartate aminotransferase, alanine aminotransferase, $\gamma$-glutamyltransferase, fasting serum lipids, serum uric acid, serum creatinine, and hemato- crit. This study also included homeostasis model assessment (HOMA) of insulin resistance (HOMA-IR) and $\beta$-cell function (HOMA- $\beta$ ). This study was approved by institutional review boards or independent ethics committees of the participating facilities, and was registered with the UMIN Clinical Trials Registry, number UMIN000031157.

\section{Statistical analysis}

The statistical analyses were performed on an intention-to-treat population. Data were presented as means \pm standard deviation (SD) or medians with the interquartile range (IQR). Statistical analyses were performed by means of appropriate parametric and nonparametric methods. Changes in continuous measures between baseline and post-treatment periods were tested by means of a paired $t$-test. Nonparametric methods were used for non-normally distributed values. Changes from baseline in UACR at week 104 were analyzed using the Wilcoxon signedrank test. The relationship between changes in UACR and blood pressure was assessed using Pearson's correlation coefficients. All analyses were performed with SPSS Statistics, version 22.0 (IBM Corp., Armonk, USA). A P-value $<0.05$ was considered statistically significant.

For the safety assessment, we determined the frequency of adverse events (AEs) and the percentage of patients affected. AEs were coded according to the Medical Dictionary for Regulatory Activities (MedDRA/J version 21.0, Japanese Maintenance Organization, Tokyo, Japan).

\section{Results}

\section{Patients}

In total, 50 patients eligible to participate in the study were treated with $50 \mathrm{mg}$ of ipragliflozin and then followed up for 24 weeks (Fig. 1). The baseline characteristics of all patients and those with diabetic nephropathy, defined as the presence of microalbuminuria (UACR $\geq 30 \mathrm{mg} / \mathrm{gCr}$ ), who entered post-trial monitoring are shown in the left and right columns, respectively, of Table 1. Forty-seven patients completed the post-trial monitoring for 104 weeks, and 43 of these patients were still being treated with ipragliflozin at 104 weeks (Fig. 1).

\section{All patients}

Table 2 shows the changes in parameters for all 50 patients receiving post-trial monitoring. In these 50 patients, the significant reductions in $\mathrm{HbAlc}$, fasting plasma glucose, and body weight observed at 24 weeks were sustained throughout the post-trial monitoring period $(\mathrm{P}<0.001, \mathrm{P}=0.020, \mathrm{P}<0.001$, respectively). Whiles the eGFRs were decreased during the intervention period, they were restored to levels similar to those at the baseline by 104 weeks. There were no significant changes in either SBP or DBP at after 104 weeks of treatment versus the baseline values (Table 2). 


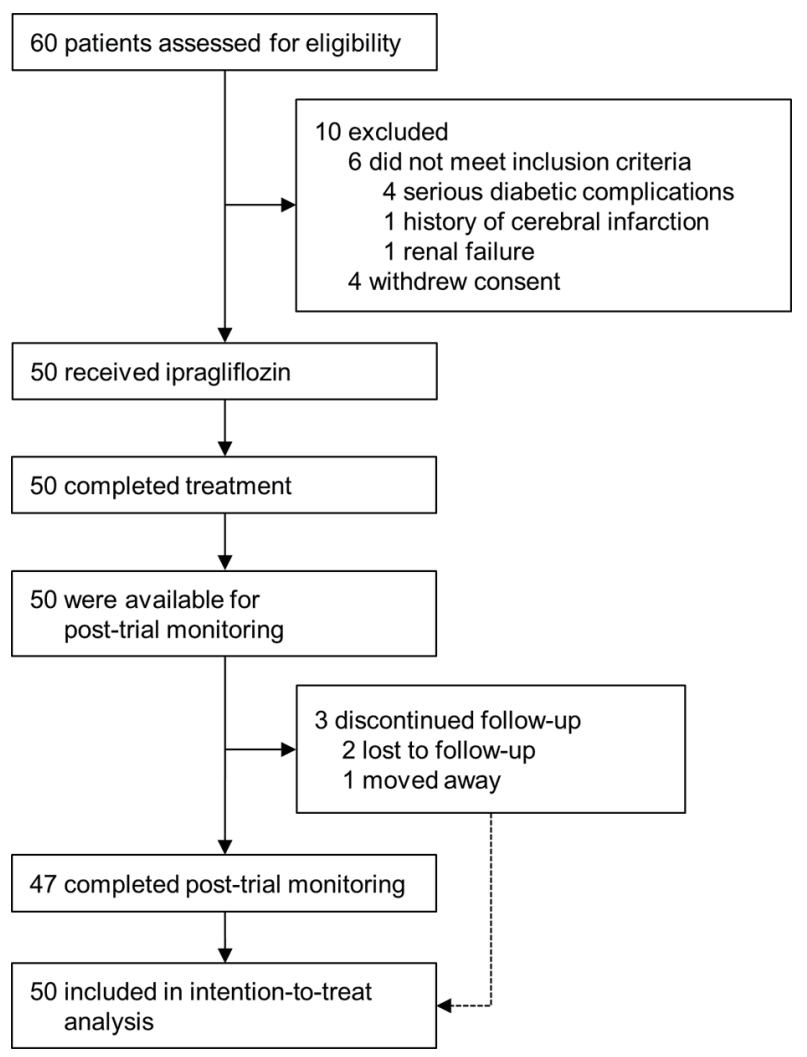

Figure 1. Enrollment and outcomes.

\section{Subgroup analysis based on renal function}

Figure 2 shows the subgroup analysis results for patients categorized according to their renal function at baseline into three groups: high $(H, e G F R \geq 90, n=20)$, medium $(M, e G F R \geq 60$ to $<90, \mathrm{n}=22$ ), and low ( $\mathrm{L}, \mathrm{eGFR}<60, \mathrm{n}=8$ ). After 24 weeks of treatment with ipragliflozin, HbA1c in the $\mathrm{H}$ and $\mathrm{M}$ groups was significantly decreased, and these significant reductions persisted until 104 weeks $(\mathrm{P}<0.001$, respectively), whereas $\mathrm{HbA} 1 \mathrm{c}$ in the L group tended to be decreased at 104 weeks but the reduction did not reach statistical significance $(\mathrm{P}=0.066)$ (Fig. 2a). The body weights of patients in all three groups remained significantly decreased through 104 weeks, regardless of baseline renal function (Fig. 2b). At 104 weeks, the median UACR had significantly decreased from $12.3(7.5$ - 89.6) at baseline to $8.2(5.6-15.7) \mathrm{mg} / \mathrm{gCr}$ only in the $\mathrm{H}$ group $(\mathrm{P}=$ 0.001 ), with no significant difference in the median UACR in either the $\mathrm{M}$ or the L group (Fig. 2d). In contrast, although the mean eGFR in the $\mathrm{H}$ group was significantly decreased at 24 weeks, this significant reduction had disappeared at 104 weeks (Fig. 2c). The $\mathrm{M}$ and $\mathrm{L}$ groups showed no significant changes in mean eGFR throughout the entire study period (Fig. 2c).

\section{Subgroup analysis based on diabetic nephropathy}

Table 3 shows the parameter changes in the 16 patients with diabetic nephropathy enrolled in post-trial monitoring between baseline and 104 weeks. After 104 weeks of ipragliflozin treatment, both mean $\mathrm{HbA} 1 \mathrm{c}$ and body weight were significantly decreased $(\mathrm{P}=0.006, \mathrm{P}<0.001$, respectively) in all patients with diabetic nephropathy. Additionally, median UACR decreased significantly from $119.2(98.9-201.8)$ at baseline to $36.9(19.7$ - 204.7) $\mathrm{mg} / \mathrm{gCr}$ at 104 weeks $(\mathrm{P}=0.011)$. In contrast, the mean eGFR did not change through 104 weeks in this subgroup.

Next, patients with diabetic nephropathy were categorized into two groups: those with high eGFR $(\mathrm{NH}, \mathrm{eGFR} \geq 80, \mathrm{n}=$ 7) and those with low eGFR (NL, eGFR < 80, n = 9) (Fig. 3). After 104 weeks, HbA1c showed a decreasing tendency and body weight was significantly decreased in both groups (Fig.

Table 1. Baseline Characteristics

\begin{tabular}{lll}
\hline & All patients & Diabetic nephropathy \\
\hline Number of patients & 50 & 16 \\
Male/female $(\mathrm{n})$ & $16 / 34$ & $6 / 10$ \\
Age (years) & $53.2 \pm 12.5$ & $53.8 \pm 13.3$ \\
Duration of diabetes (years) & $9.1 \pm 6.0$ & $9.9 \pm 7.2$ \\
Body mass index $\left(\mathrm{kg} / \mathrm{m}^{2}\right)$ & $30.6 \pm 6.2$ & $32.1 \pm 4.5$ \\
Fasting C-peptide $(\mathrm{ng} / \mathrm{mL})$ & $3.4 \pm 1.8$ & $3.5 \pm 1.03$ \\
Medications & & $10(62.5 \%)$ \\
$\quad$ Metformin $(\%)$ & $31(62.0 \%)$ & $8(50.0 \%)$ \\
$\quad 31(62.0 \%)$ & $2(12.5 \%)$ \\
$\quad$ DPP-4 inhibitor $(\%)$ & $10(20.0 \%)$ & $5(31.3 \%)$ \\
Insulin $(\%)$ & $12(24.0 \%)$ & $13(81.3 \%)$ \\
ARB or ACE inhibitor $(\%)$ & $32(64.0 \%)$ & $10(62.5 \%)$ \\
\hline Statin $(\%)$ & $32(64.0 \%)$ & \\
\hline
\end{tabular}

Data are $\mathrm{n}(\%)$, means \pm standard deviation (SD). DPP-4: dipeptidyl peptidase-4; ARB: angiotensin II receptor blocker; ACE: angiotensin-converting enzyme. 
Table 2. Changes in the Parameters of All Patients Between Baseline and Week $104(n=50)$

\begin{tabular}{|c|c|c|c|c|c|c|}
\hline & \multicolumn{2}{|c|}{ Interventional trial period } & \multirow{2}{*}{$\begin{array}{l}\text { P value (week } \\
24 \text { vs. baseline) }\end{array}$} & \multicolumn{2}{|c|}{ Post-trial monitoring period } & \multirow{2}{*}{$\begin{array}{l}\text { P value (week } \\
104 \text { vs. baseline) }\end{array}$} \\
\hline & Baseline & Week 24 & & Week 52 & Week 104 & \\
\hline $\mathrm{HbA} 1 \mathrm{c}(\%)$ & $8.7 \pm 1.5$ & $7.7 \pm 1.2$ & $<0.001$ & $7.7 \pm 1.3$ & $7.6 \pm 1.2$ & $<0.001$ \\
\hline Body weight (kg) & $78.4 \pm 19.7$ & $75.8 \pm 20.1$ & $<0.001$ & $76.0 \pm 20.1$ & $76.5 \pm 20.6$ & $<0.001$ \\
\hline $\mathrm{UACR}(\mathrm{mg} / \mathrm{gCr})$ & $15.5(8.0-95.7)$ & $12.9(7.4-36.3)$ & $0.011^{\dagger}$ & $15.2(8.0-39.0)$ & $14.0(7.3-34.6)$ & $0.053^{\dagger}$ \\
\hline DBP (mm Hg) & $80.7 \pm 11.0$ & $80.8 \pm 10.5$ & 0.888 & $80.9 \pm 12.9$ & $79.4 \pm 10.9$ & 0.411 \\
\hline Fasting serum insulin $(\mu \mathrm{U} / \mathrm{mL})$ & $13.4 \pm 6.3$ & $13.2 \pm 11.5$ & 0.924 & $13.4 \pm 11.7$ & $12.9 \pm 11.2$ & 0.745 \\
\hline HOMA-IR & $5.5 \pm 3.4$ & $4.7 \pm 5.0$ & 0.378 & $5.0 \pm 5.0$ & $4.7 \pm 4.8$ & 0.369 \\
\hline НOMA- $\beta(\%)$ & $54.7 \pm 35.3$ & $67.0 \pm 44.0$ & 0.064 & $62.4 \pm 45.4$ & $61.1 \pm 42.9$ & 0.336 \\
\hline Triglycerides (mg/dL) & $157.1 \pm 77.3$ & $134.1 \pm 71.7$ & 0.028 & $149.1 \pm 80.7$ & $159.8 \pm 98.3$ & 0.849 \\
\hline AST (IU/L) & $39.4 \pm 16.7$ & $26.0 \pm 8.6$ & $<0.001$ & $28.2 \pm 11.8$ & $33.1 \pm 19.7$ & 0.044 \\
\hline ALT (IU/L) & $56.2 \pm 29.7$ & $35.6 \pm 19.5$ & $<0.001$ & $38.8 \pm 21.0$ & $44.6 \pm 33.4$ & 0.003 \\
\hline$\gamma-\mathrm{GT}(\mathrm{IU} / \mathrm{L})$ & $62.3 \pm 55.6$ & $41.0 \pm 34.5$ & $<0.001$ & $45.1 \pm 57.5$ & $54.0 \pm 84.0$ & 0.396 \\
\hline Uric acid (mg/dL) & $5.5 \pm 1.3$ & $4.7 \pm 1.1$ & $<0.001$ & $4.9 \pm 1.2$ & $4.9 \pm 1.3$ & $<0.001$ \\
\hline Serum creatinine $(\mathrm{mg} / \mathrm{dL})$ & $0.68 \pm 0.16$ & $0.71 \pm 0.16$ & 0.011 & $0.68 \pm 0.16$ & $0.68 \pm 0.17$ & 0.721 \\
\hline Hematocrit (\%) & $42.6 \pm 4.1$ & $45.4 \pm 4.5$ & $<0.001$ & $44.8 \pm 4.8$ & $45.5 \pm 4.3$ & $<0.001$ \\
\hline
\end{tabular}

Data are means \pm standard deviation (SD) or medians with the interquartile range (IQR). P value versus baseline by the paired $t$-test. ${ }^{t} \mathrm{P}$ value versus baseline by the Wilcoxon signed-rank test. HbA1c: glycated hemoglobin; UACR: urinary albumin-to-creatinine ratio; eGFR: estimated glomerular filtration rate; SBP: systolic blood pressure; DBP: diastolic blood pressure; HOMA-IR: homeostasis model assessment of insulin resistance; HOMA- $\beta$ : homeostasis model assessment of $\beta$-cell function; LDL-cholesterol: low-density lipoprotein cholesterol; HDL-cholesterol: high-density lipoprotein cholesterol; AST: aspartate aminotransferase; ALT: alanine aminotransferase; $\gamma$-GT: $\gamma$ - glutamyltransferase.

3a, b). Importantly, we observed highly favorable, clinically, and statistically significant decreases in median UACR from 106.7 (92.3 - 274.3) at baseline to $26.7(18.2$ - 127.1) $\mathrm{mg} / \mathrm{gCr}$ at 104 weeks only in the NH group ( $\mathrm{P}=0.018)$ (Fig. 3d). In contrast, the decreases observed in median UACR in the NL group did not reach statistical significance (Fig. 3d). The mean eGFR did not change through 104 weeks in this subgroup, consistent with the results of the analysis of all patients (Fig. 3c).

\section{Analysis of blood pressure}

There were no significant changes in blood pressure levels in any of the patients enrolled in this study during the 104-week observation period. Next, subgroup analysis of 23 patients with poorly controlled blood pressure, defined as an SBP $\geq$ $140 \mathrm{~mm} \mathrm{Hg}$ and/or a DBP $\geq 90 \mathrm{~mm} \mathrm{Hg}$, showed significant decreases in the values of both SBP and DBP following 24 weeks of ipragliflozin treatment ( $\mathrm{P}=0.035, \mathrm{P}=0.045$, respectively). Although SBP and DBP values at the end of 104 weeks, as compared to baseline, were decreased $(141.3 \pm 9.8$ vs. $136.4 \pm$ $9.7 \mathrm{~mm} \mathrm{Hg} ; 88.1 \pm 9.2$ vs. $85.7 \pm 9.0 \mathrm{~mm} \mathrm{Hg}$, respectively), the reductions did not reach statistical significance.
In contrast, analysis of the relationship between the amount of change in UACR and blood pressure at 24 weeks after the start of treatment revealed a significant positive correlation between UACR and SBP values, independently of the presence of diabetic nephropathy. However, these significant correlations had disappeared by 104 weeks (Table 4).

\section{Additional efficacy evaluations}

Tables 2 and 3 show changes observed in other efficacy parameters after 104 weeks of ipragliflozin treatment. Significant improvements in serum lipids, hepatic function, and uric acid values observed during the interventional trial period in all patients were sustained throughout the post-trial monitoring period. We also noted a significantly increased mean hematocrit level in all patients, regardless of the presence of diabetic nephropathy (Tables 2 and 3).

\section{Safety}

During the 104-week observation period, AEs occurred in 10 
a

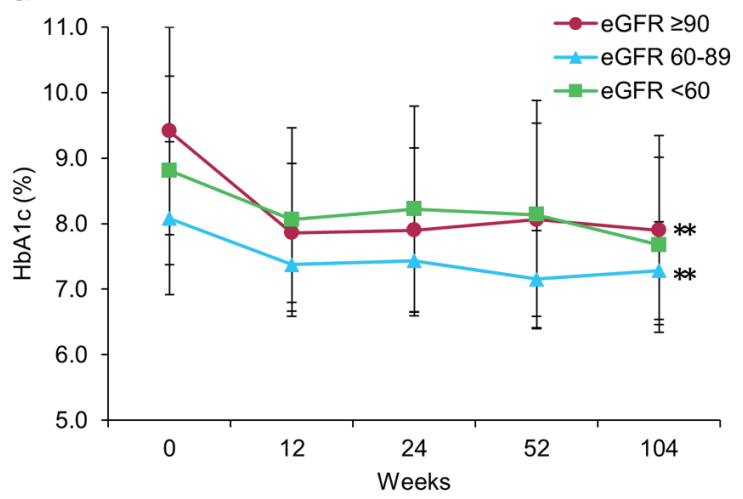

C

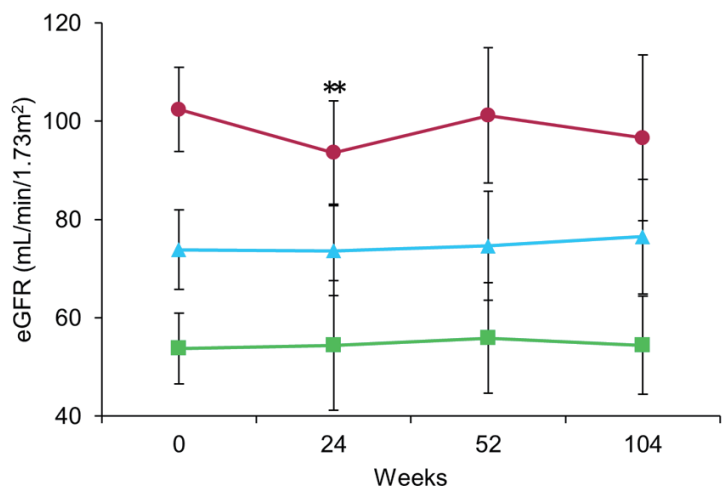

b

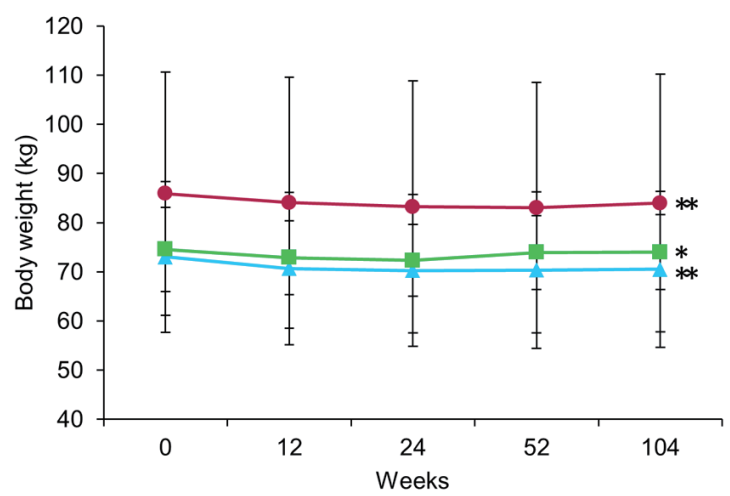

d

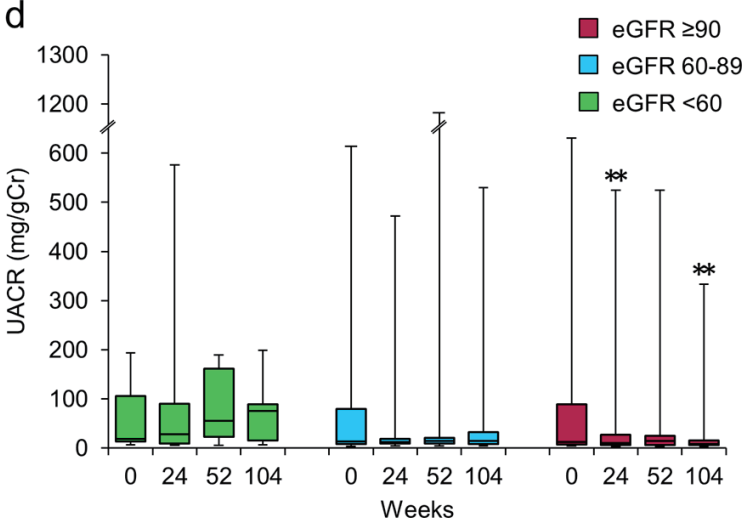

Figure 2. Effects of ipragliflozin on efficacy parameters at week 104 in all patients $(n=50)$, classified according to estimated glomerular filtration rate (eGFR) at baseline into three groups; eGFR $\geq 90$ (circles; $n=20$ ), eGFR $\geq 60$ to $<90$ (triangles; $n=22$ ), and eGFR < 60 (squares; $n=8$ ). (a) Change from baseline in glycated hemoglobin (HbA1c) to week 104 after starting ipragliflozin administration. (b) Change from baseline in body weight. (c) Change from baseline in eGFR. (d) Change from baseline in urinary albumin-to-creatinine ratio (UACR) at week 104. Data are means \pm standard deviation (SD) or medians with the interquartile range (IQR). ${ }^{*} \mathrm{P}<0.05,{ }^{* *} \mathrm{P}<0.01$ versus baseline by the paired $t$-test. For the UACR, the Wilcoxon signed-rank test was used to evaluate changes between the baseline and week 104. Error bars show SDs or IQRs.

subjects $(20.0 \%)$. The AEs included urinary tract infection in two subjects $(4.0 \%)$, vulvovaginal candidiasis in two $(4.0 \%)$, diarrhea in four $(8.0 \%)$, and hyperphagia in two $(4.0 \%)$. These adverse effects resolved in response to administration of appropriate antibiotics or antifungals, while one patient with hyperphagia discontinued ipragliflozin treatment on the advice of a physician. There were no other AEs such as severe hypoglycemia, dehydration, ketoacidosis, cardiac failure or severe infections, necessitating treatment interruption or termination of ipragliflozin administration.

\section{Discussion}

In the post-trial monitoring study, we demonstrated that ipragliflozin significantly reduced urinary albumin excretion in patients with type 2 diabetes without decreasing eGFR for 104 weeks. In addition, sustained improvements in glycemic control and body weight reductions were observed during the 104 weeks of the post-trial follow-up in a subset of patients with type 2 diabetes. The present study showed ipragliflozin to exert pleiotropic effects on glycemic control, body weight, blood pressure, and diabetic nephropathy in a real-world setting. However, this was a small study, making it difficult to draw definitive conclusions regarding ipragliflozin treatment.

In recent years, clinical and experimental studies have shown that SGLT2 inhibitors contribute to the prevention of diabetic nephropathy progression. In the EMPA-REG OUTCOME trial, empagliflozin reduced the risk of major adverse cardiovascular events and was associated with slower progression of kidney disease and lower rates of clinically relevant renal events in patients with type 2 diabetes at high risk for cardiovascular events $[9,10]$. Similarly, the CANVAS Program trial reported that patients with type 2 diabetes and an elevated risk of cardiovascular disease treated with canagliflozin had a lower risk of cardiovascular and renal events [12]. In addition, several clinical and experimental studies have shown that SGLT2 inhibitors exert favorable effects preventing diabetic kidney disease progression [13-18].

The analysis of renal hemodynamic effects of SGLT2 inhibition demonstrated that empagliflozin attenuated renal hyperfiltration in patients with type 1 diabetes under clamped euglycemic and hyperglycemic conditions, through improvement of tubular-glomerular feedback mechanisms [6]. In addi- 
Table 3. Changes in the Parameters of Patients With Diabetic Nephropathy Between Baseline and Week $104(\mathrm{n}=16)$

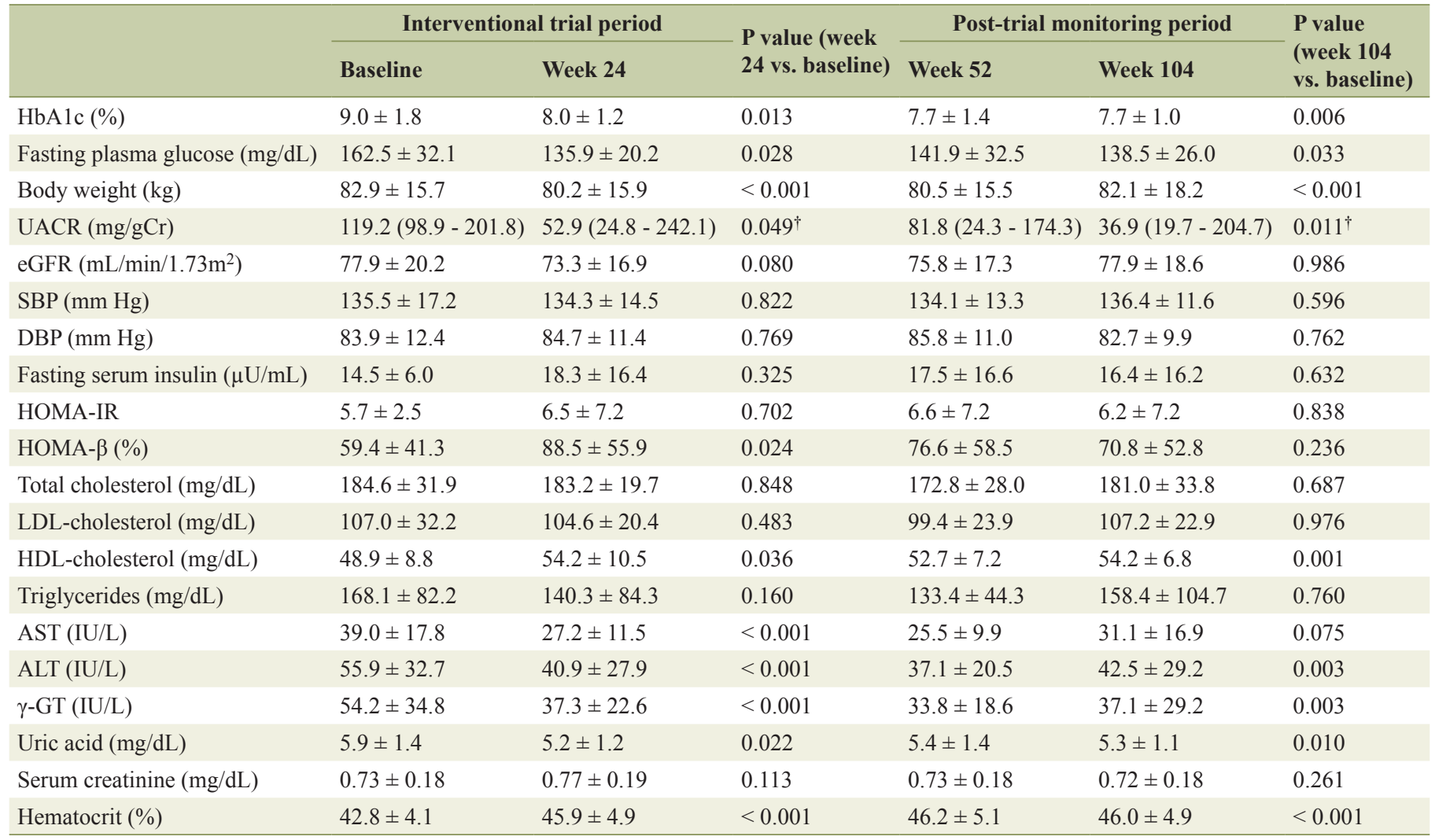

Data are means \pm standard deviation (SD) or medians with the interquartile range (IQR). $\mathrm{P}$ value versus baseline by the paired $t$-test. ${ }^{t} \mathrm{P}$ value versus baseline by the Wilcoxon signed-rank test. HbA1c: glycated hemoglobin; UACR: urinary albumin-to-creatinine ratio; eGFR: estimated glomerular filtration rate; SBP: systolic blood pressure; DBP: diastolic blood pressure; HOMA-IR: homeostasis model assessment of insulin resistance; HOMA- $\beta$ : homeostasis model assessment of $\beta$-cell function; LDL-cholesterol: low-density lipoprotein cholesterol; HDL-cholesterol: high-density lipoprotein cholesterol; AST: aspartate aminotransferase; ALT: alanine aminotransferase; $\gamma$-GT: $\gamma$ - glutamyltransferase.

tion, empagliflozin was found to improve glomerular hyperfiltration independently of blood glucose in type 1 diabetic Akita mice, and was reported to suppress the renal expressions of inflammatory genes, urinary albumin excretion, and kidney weight [16]. These findings suggest the main mechanism by which SGLT2 inhibitors attenuate diabetic nephropathy to be amelioration of glomerular hyperfiltration. In fact, in our previous study, ipragliflozin significantly reduced urinary albumin excretion and ameliorated glomerular hyperfiltration in patients with type 2 diabetes whose renal functions were relatively maintained. In contrast, although $\mathrm{HbA}$ 1c reductions persisted for 104 weeks, this significant amelioration of glomerular hyperfiltration was no longer present at the end of the post-trial monitoring period. In addition, a significant reduction of uncontrolled blood pressure and a positive correlation between UACR and SBP changes, as observed during the interventional trial period in our previous study, were also no longer present at the end of the post-trial monitoring period. Despite the loss of these benefits in the interventional trial period, the UACR reduction persisted through 104 weeks. These results indicate that the mechanisms by which SGLT2 inhibitors attenuate diabetic nephropathy are attributable, not only to improvements in glycemic control, glomerular hyperfiltration and blood pressure, but also to other favorable mechanisms. For example, an experimental study using mice fed a highfat diet demonstrated that SGLT2 inhibition by ipragliflozin might act directly on tubular cells and protect kidney tubular cells from mitochondrial damage due to metabolic insults regardless of blood glucose levels or favorable body weight reductions [19]. Furthermore, Kamezaki et al recently reported that even low-dose ipragliflozin, given to type 2 diabetic mice, reduced renal cortical hypoxia and abnormal hemodynamics in early diabetic nephropathy. In addition to these effects, highdose ipragliflozin exerted renoprotective effects by reducing oxidative stress in tubular epithelia and glomerular podocytes [20]. These prior studies demonstrated that SGLT2 inhibitors exert renoprotective effects by having beneficial effects not only on glycemic control, glomerular hyperfiltration, blood pressure, and body weight reduction, but also through multifactorial mechanisms.

Currently, all six SGLT2 inhibitors are commercially available in Japan, and their pleiotropic effects on various complications appear to be very similar. However, the pharmacokinetic and pharmacodynamic properties of all six SGLT2 inhibitors were investigated and compared in an experimental study using normal and diabetic mice, and it was found that 
a

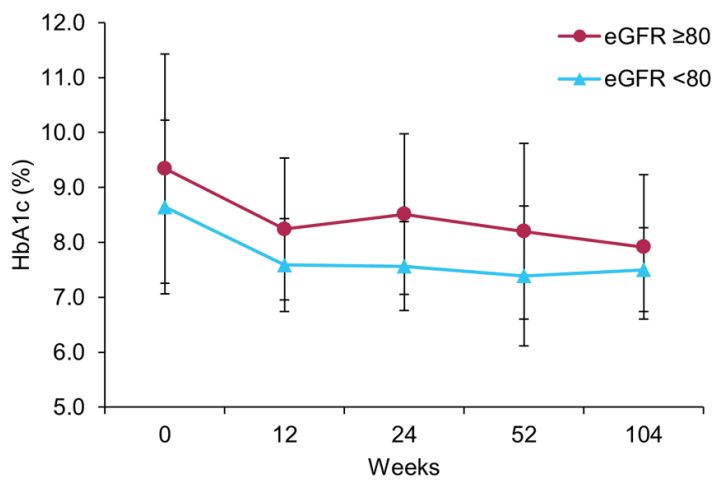

C

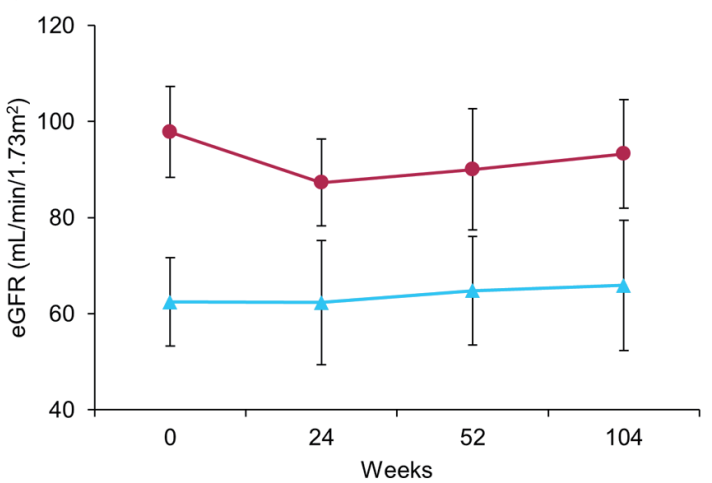

b

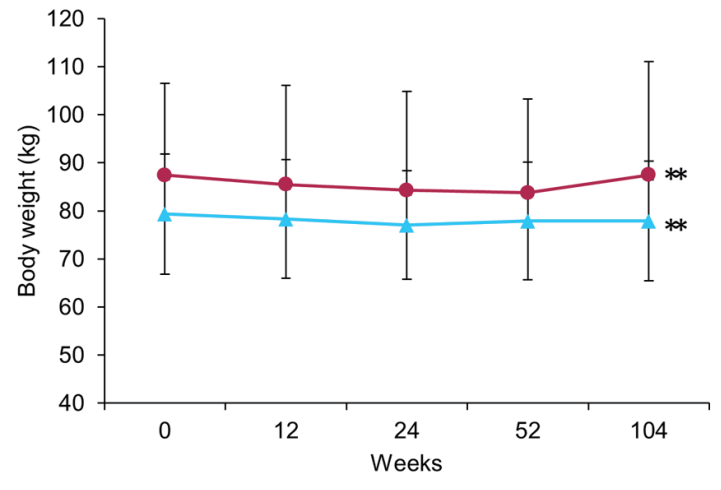

d

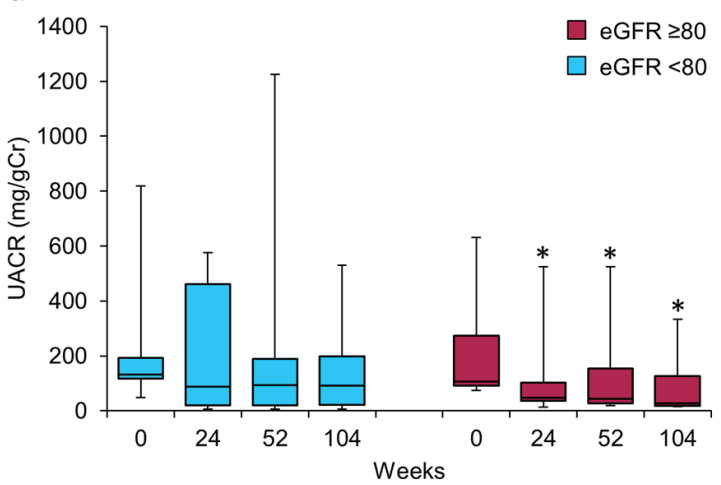

Figure 3. Effects of ipragliflozin on efficacy parameters at week 104 in patients with diabetic nephropathy $(n=16)$, classified according to estimated glomerular filtration rate (eGFR) at baseline into two groups; eGFR $\geq 80$ (circles; $n=7$ ) and eGFR < 80 (triangles; $n=9$ ). (a) Change from baseline in glycated hemoglobin (HbA1c) to week 104 after starting ipragliflozin administration. (b) Change from baseline in body weight. (c) Change from baseline in eGFR. (d) Change from baseline in urinary albumin-tocreatinine ratio (UACR) at week 104. Data are means \pm standard deviation (SD) or medians with the interquartile range (IQR). ${ }^{*} \mathrm{P}<0.05,{ }^{* *} \mathrm{P}<0.01$ versus baseline by the paired $t$-test. For the UACR, the Wilcoxon signed-rank test was used to evaluate changes between the baseline and week 104. Error bars show SDs or IQRs.

the ameliorating effects on features of type 2 diabetes differ slightly, in terms of both the durations and the onsets of pharmacologic effects, among these SGLT2 inhibitors [21]. In the present study, ipragliflozin was classified into a category of long-acting agents, exhibiting effects superior to those of other SGLT2 inhibitors with respect to the rapid onset of pharmacological effects. The duration and onset of these pharmacologic effects appeared to correlate closely with the pharmacokinetic properties specific to each SGLT2 inhibitor, particularly with respect to high distribution and long retention in the target organ, i.e. the kidney [21]. Therefore, focusing on the pharmacokinetic and pharmacodynamic properties, we can reasonably assume that beneficial effects for various complications, particularly in patients with diabetic nephropathy, differ slightly

Table 4. Correlations Among UACR and Blood Pressure, HbA1c, and Body Weight

\begin{tabular}{|c|c|c|c|c|c|c|c|c|}
\hline & \multicolumn{4}{|c|}{ Interventional trial period (week 24) } & \multicolumn{4}{|c|}{ Post-trial monitoring period (week 104) } \\
\hline & \multirow{2}{*}{\multicolumn{2}{|c|}{$\begin{array}{c}\text { All patients }(\mathrm{n}=50) \\
\Delta \mathrm{UACR}(\mathrm{mg} / \mathrm{gCr})\end{array}$}} & \multirow{2}{*}{\multicolumn{2}{|c|}{$\begin{array}{c}\text { Diabetic nephropathy }(\mathrm{n}=16) \\
\Delta \mathrm{UACR}(\mathrm{mg} / \mathrm{gCr})\end{array}$}} & \multirow{2}{*}{\multicolumn{2}{|c|}{$\begin{array}{c}\text { All patients }(\mathrm{n}=50) \\
\Delta \mathrm{UACR}(\mathrm{mg} / \mathrm{gCr}) \\
\end{array}$}} & \multirow{2}{*}{\multicolumn{2}{|c|}{$\begin{array}{c}\text { Diabetic nephropathy }(\mathrm{n}=16) \\
\Delta \mathrm{UACR}(\mathrm{mg} / \mathrm{gCr})\end{array}$}} \\
\hline & & & & & & & & \\
\hline$\triangle \mathrm{SBP}(\mathrm{mm} \mathrm{Hg})$ & 0.342 & 0.015 & 0.504 & 0.047 & 0.074 & 0.619 & 0.140 & 0.606 \\
\hline$\triangle \mathrm{DBP}(\mathrm{mm} \mathrm{Hg})$ & 0.110 & 0.445 & 0.148 & 0.585 & 0.057 & 0.698 & 0.044 & 0.870 \\
\hline
\end{tabular}

The relationship between changes in UACR and blood pressure, HbA1c, and body weight were assessed using Pearson's correlation coefficients. $\Delta$ : the amount of change week 24 or week 104 after baseline. UACR: urinary albumin-to-creatinine ratio; HbA1c: glycated hemoglobin; SBP: systolic blood pressure; DBP: diastolic blood pressure; BW: body weight. 
among SGLT2 inhibitors.

In contrast, in an exploratory mediation analysis derived from the EMPA-REG OUTCOME trial, changes in hematocrit and hemoglobin were the most important mediators of the reduction in risk of cardiovascular death with empagliflozin versus placebo, rather than changes in $\mathrm{HbAlc}$, body weight, or UACR [22]. Enhanced erythropoiesis could be a complementary mechanism to the hemodynamic changes reflected by increases in hematocrit and hemoglobin in patients treated with SGLT2 inhibitors [22]. In patients with diabetes, the proximal tubules are overtaxed by excessive glucose reabsorption and the resulting increased oxygen requirement causes tubulointerstitial hypoxia [23]. SGLT2 inhibitors reduce the workload of the proximal tubules and improve tubulointerstitial hypoxia and accordingly restore normal erythropoietin production. In fact, in our present study, we actually observed significantly increased hematocrit, regardless of the presence of diabetic nephropathy. This result suggests that an increase in hematocrit in response to treatment with SGLT2 inhibitors may reflect suppression or slowing of diabetic nephropathy progression, thereby serving as a predictor of the outcomes of routine medical practices. Consequently, improvement of tubulointerstitial hypoxia with SGLT2 inhibitor treatment might contribute, not only to improving diabetic kidney disease, but also to the observed cardiovascular benefits [24].

On the other hand, the renoprotective effects exerted by another class of glucose lowering drugs have also been reported. For example, an experimental study demonstrated that the dipeptidyl peptidase (DPP)-4 inhibitor linagliptin showed efficacy comparable to that of the angiotensin II receptor blocker telmisartan in preventing chronic kidney disease progression in non-diabetic rats with 5/6 nephrectomy [25]. Furthermore, preclinical animal studies as well as clinical data have raised the possibility that DPP-4 inhibitors decrease the progression of diabetic nephropathy in a blood pressure- and glucose-independent manner [26]. These studies showed the renoprotective effects of the drugs studied to be attributable to an increased half-life of DPP-4 substrates such as glucagon-like peptide (GLP)-1.

In addition, a pre-specified analysis of the LEADER trial revealed that the GLP-1 receptor agonist liraglutide reduced both the rate of development and the progression of diabetic kidney disease as compared with a placebo in 9,340 patients with type 2 diabetes and high cardiovascular risk [27]. Although the demonstration of beneficial renal effects of both GLP-1 receptor agonists and SGLT2 inhibitors represents a breakthrough in the treatment of diabetic nephropathy, SGLT2 inhibitors might have greater potency than GLP-1 receptor agonists with respect to kidney protection. A comparison of the EMPA-REG OUTCOME and LEADER trials demonstrated empagliflozin to be associated with slower progression of kidney disease and lower rates of clinically relevant renal events, including progression to macroalbuminuria, doubling of the serum creatinine level, initiation of renal-replacement therapy, and death from renal disease [10]. By contrast, liraglutide reduced new onset of persistent macroalbuminuria, but had no significant effect on other renal outcomes [28]. These results suggest that SGLT2 inhibitors contribute to the prevention of diabetic nephropathy progression by mechanisms that clearly differ from those of other antidiabetic drugs. We advocate that future studies focus on elucidating the details of the differences in renoprotective effects between SGLT2 inhibitors and other drugs.

In conclusion, the present study confirmed ipragliflozin to significantly reduce urinary albumin excretion in patients with diabetic nephropathy without decreasing eGFR for 104 weeks. In addition, sustained improvements in glycemic control and body weight reductions were observed during the 104-week post-trial follow-up. However, this study has limitations. The number of subjects was too small to allow definitive conclusions about the investigated drug to be drawn. Furthermore, the study was performed at only a small number of facilities and a single-arm design was used. As stated above, SGLT2 inhibitors have pleiotropic effects on diabetic kidney disease. In the future, we hope to investigate these mechanisms in greater detail and to optimize the use of SGLT2 inhibitors for more patients.

\section{Acknowledgments}

No financial support for this study was provided by any company or foundation. We thank all investigators, trial staff, and participants.

\section{Conflict of Interest}

The authors declare that they have no conflict of interest concerning this article.

\section{Grant Support}

None.

\section{References}

1. Gallo LA, Wright EM, Vallon V. Probing SGLT2 as a therapeutic target for diabetes: basic physiology and consequences. Diab Vasc Dis Res. 2015;12(2):78-89.

2. Vasilakou D, Karagiannis T, Athanasiadou E, Mainou M, Liakos A, Bekiari E, Sarigianni M, et al. Sodiumglucose cotransporter 2 inhibitors for type 2 diabetes: a systematic review and meta-analysis. Ann Intern Med. 2013;159(4):262-274.

3. Iizuka T, Iemitsu $\mathrm{K}$, Takihata $\mathrm{M}$, Takai M, Nakajima S, Minami N, Umezawa S, et al. Efficacy and safety of ipragliflozin in Japanese patients with type 2 diabetes: interim outcome of the ASSIGN-K study. J Clin Med Res. 2016;8(2):116-125.

4. Iemitsu K, Iizuka T, Takihata M, Takai M, Nakajima S, Minami N, Umezawa S, et al. Factors influencing changes in hemoglobin a1c and body weight during treatment of type 2 diabetes with ipragliflozin: interim analysis of the ASSIGN-K study. J Clin Med Res. 2016;8(5):373- 
378

5. Baker WL, Smyth LR, Riche DM, Bourret EM, Chamberlin KW, White WB. Effects of sodium-glucose co-transporter 2 inhibitors on blood pressure: a systematic review and meta-analysis. J Am Soc Hypertens. 2014;8(4):262275 e269.

6. Cherney DZ, Perkins BA, Soleymanlou N, Maione M, Lai V, Lee A, Fagan NM, et al. Renal hemodynamic effect of sodium-glucose cotransporter 2 inhibition in patients with type 1 diabetes mellitus. Circulation. 2014;129(5):587597.

7. Ito D, Shimizu S, Inoue K, Saito D, Yanagisawa M, Inukai K, Akiyama Y, et al. Comparison of ipragliflozin and pioglitazone effects on nonalcoholic fatty liver disease in patients with type 2 diabetes: a randomized, 24week, open-label, active-controlled trial. Diabetes Care. 2017;40(10):1364-1372.

8. Wu JH, Foote C, Blomster J, Toyama T, Perkovic V, Sundstrom J, Neal B. Effects of sodium-glucose cotransporter-2 inhibitors on cardiovascular events, death, and major safety outcomes in adults with type 2 diabetes: a systematic review and meta-analysis. Lancet Diabetes Endocrinol. 2016;4(5):411-419.

9. Zinman B, Wanner C, Lachin JM, Fitchett D, Bluhmki E, Hantel S, Mattheus M, et al. Empagliflozin, cardiovascular outcomes, and mortality in type 2 diabetes. N Engl J Med. 2015;373(22):2117-2128.

10. Wanner C, Inzucchi SE, Lachin JM, Fitchett D, von Eynatten M, Mattheus M, Johansen OE, et al. Empagliflozin and progression of kidney disease in type 2 diabetes. N Engl J Med. 2016;375(4):323-334.

11. Ito D, Ikuma-Suwa E, Inoue K, Kaneko K, Yanagisawa $\mathrm{M}$, Inukai K, Noda M, et al. Effects of ipragliflozin on diabetic nephropathy and blood pressure in patients with type 2 diabetes: an open-label study. J Clin Med Res. 2017;9(2):154-162.

12. Neal B, Perkovic V, Mahaffey KW, de Zeeuw D, Fulcher G, Erondu N, Shaw W, et al. Canagliflozin and cardiovascular and renal events in type 2 diabetes. N Engl J Med. 2017;377(7):644-657.

13. Cherney D, Lund SS, Perkins BA, Groop PH, Cooper ME, Kaspers S, Pfarr E, et al. The effect of sodium glucose cotransporter 2 inhibition with empagliflozin on microalbuminuria and macroalbuminuria in patients with type 2 diabetes. Diabetologia. 2016;59(9):1860-1870.

14. Heerspink HJ, Johnsson E, Gause-Nilsson I, Cain VA, Sjostrom CD. Dapagliflozin reduces albuminuria in patients with diabetes and hypertension receiving renin-angiotensin blockers. Diabetes Obes Metab. 2016;18(6):590-597.

15. Gilbert RE. Sodium-glucose linked transporter-2 inhibitors: potential for renoprotection beyond blood glucose lowering? Kidney Int. 2014;86(4):693-700.

16. Vallon V, Gerasimova M, Rose MA, Masuda T, Satriano J, Mayoux E, Koepsell H, et al. SGLT2 inhibitor empagli- flozin reduces renal growth and albuminuria in proportion to hyperglycemia and prevents glomerular hyperfiltration in diabetic Akita mice. Am J Physiol Renal Physiol. 2014;306(2):F194-204.

17. Vallon V, Rose M, Gerasimova M, Satriano J, Platt KA, Koepsell H, Cunard R, et al. Knockout of Na-glucose transporter SGLT2 attenuates hyperglycemia and glomerular hyperfiltration but not kidney growth or injury in diabetes mellitus. Am J Physiol Renal Physiol. 2013;304(2):F156-167.

18. Terami N, Ogawa D, Tachibana H, Hatanaka T, Wada J, Nakatsuka A, Eguchi J, et al. Long-term treatment with the sodium glucose cotransporter 2 inhibitor, dapagliflozin, ameliorates glucose homeostasis and diabetic nephropathy in db/db mice. PLoS One. 2014;9(6):e100777.

19. Takagi S, Li J, Takagaki Y, Kitada M, Nitta K, Takasu T, Kanasaki K, et al. Ipragliflozin improves mitochondrial abnormalities in renal tubules induced by a high-fat diet. J Diabetes Investig. 2018.

20. Kamezaki M, Kusaba T, Komaki K, Fushimura Y, Watanabe N, Ikeda K, Kitani T, et al. Comprehensive renoprotective effects of ipragliflozin on early diabetic nephropathy in mice. Sci Rep. 2018;8(1):4029.

21. Tahara A, Takasu T, Yokono M, Imamura M, Kurosaki E. Characterization and comparison of sodium-glucose cotransporter 2 inhibitors in pharmacokinetics, pharmacodynamics, and pharmacologic effects. J Pharmacol Sci. 2016;130(3):159-169.

22. Inzucchi SE, Zinman B, Fitchett $D$, Wanner C, Ferrannini E, Schumacher M, Schmoor C, et al. How does empagliflozin reduce cardiovascular mortality? Insights from a mediation analysis of the EMPA-REG OUTCOME Trial. Diabetes Care. 2018;41(2):356-363.

23. Sano M, Takei M, Shiraishi Y, Suzuki Y. Increased hematocrit during sodium-glucose cotransporter 2 inhibitor therapy indicates recovery of tubulointerstitial function in diabetic kidneys. J Clin Med Res. 2016;8(12):844-847.

24. Sano M. Hemodynamic effects of sodium-glucose cotransporter 2 inhibitors. J Clin Med Res. 2017;9(6):457-460.

25. Tsuprykov O, Ando R, Reichetzeder C, von Websky K, Antonenko V, Sharkovska Y, Chaykovska L, et al. The dipeptidyl peptidase inhibitor linagliptin and the angiotensin II receptor blocker telmisartan show renal benefit by different pathways in rats with 5/6 nephrectomy. Kidney Int. 2016;89(5):1049-1061.

26. Hasan AA, Hocher B. Role of soluble and membranebound dipeptidyl peptidase-4 in diabetic nephropathy. J Mol Endocrinol. 2017;59(1):R1-R10.

27. Mann JFE, Orsted DD, Brown-Frandsen K, Marso SP, Poulter NR, Rasmussen S, Tornoe K, et al. Liraglutide and renal outcomes in type 2 diabetes. N Engl J Med. 2017;377(9):839-848.

28. Hocher B, Tsuprykov O. Diabetic nephropathy: Renoprotective effects of GLP1R agonists and SGLT2 inhibitors. Nat Rev Nephrol. 2017;13(12):728-730. 